The Summer Undergraduate Research Fellowship (SURF) Symposium

4 August 2016

Purdue University, West Lafayette, Indiana, USA

\title{
Investigation of Aluminum Foams and Graphite Fillers for Improving the Thermal Conductivity of Paraffin Wax-based Phase Change Materials
}

Javieradrian Ruiz, Yash Gantara, Alex Bruce, John Howarter, and Amy Marconnet

\begin{abstract}
:
Passive thermal management with phase change materials (PCMs) has become the one of the most promising methods to cool cell phone processors due to the relatively simple implementation and profound impact on processor temperatures. Enhancing the thermal properties of conventional PCMs, mainly thermal conductivity and latent heat storage, allows for an overall improved thermal management system. This study aims to improve the thermal conductivity of paraffin wax (a typical commercial PCM) by the introduction of an expanded graphite (EG) filler to form a paraffin wax composite, and then infiltration of the EG/paraffin composite into an aluminum foam matrix. The thermal conductivity of the EG/paraffin composites increases respectively to the percentage by volume of expanded graphite. While the thermal conductivity increased, there is some negative impact on latent heat storage compared to pure paraffin wax. The pore size of the aluminum foam matrixes also has a profound impact on both thermal conductivity and latent heat storage of the overall system. These results will allow for improvements in cooling techniques incorporated within cell phones and other mobile devices, allowing for future development of their processors (higher computational power), prolonged reliability, and longer anticipated life cycles.
\end{abstract}

\section{Keywords}

Passive thermal management, Phase change materials (PCMs), Thermal conductivity enhancement, Thermal management of electronics 